\title{
Can the Beast Be Tamed? The Woeful Tale of Accurate Health Information on the Internet
}

\author{
J. Leonard Lichtenfeld, MD, MACP
}

Office of the Deputy Chief Medical Officer, National Home Office, American Cancer Society, Atlanta, GA

The article by Lawrentschuk et al. ${ }^{1}$ in this issue of the Annals of Surgical Oncology highlights the promise and the problems associated with medical information on the Internet.

Although the Internet is a powerful tool, the sad reality is that much of the information fails to meet a generally accepted standard for medical information-namely the World Health Organization Health on the Net (HON) standards, ${ }^{2}$ which are a reasonable measure of the commitment of medical Web sites to provide quality information regarding cancer, among other diseases, and its treatment.

The problems are not new.

For decades, physicians have struggled with the quality of information available to patients as presented through various forms of media. In the past, it was limited to reporting in newspapers, magazines, and television. Now, the issue has grown geometrically with the Internet and is no longer limited to what may be available in a particular locale. The content and the audience is international in scope, and the volume of material accessible to patients, their families, and those concerned about their disease and care has no limits. It is in fact a modern-day version of the American Wild West, where almost anything goes. You don't have to be credible, knowledgeable, or have appropriate experience to have your thoughts posted on a Web site or a chat room. There is no lack of people who are willing to provide advice and counsel to cancer patients, without any limitation on the quality of that information or the credentials of those who offer their wisdom (or lack thereof).

As pointed out by the authors, the sad reality is that the measureable quality of cancer-related information on the

(C) Society of Surgical Oncology 2011

Published Online: 23 November 2011

J. Leonard Lichtenfeld, MD, MACP

e-mail: Len.Lichtenfeld@cancer.org
Internet is woefully short of needs and expectations. A small minority of cancer-related Web sites are HON accredited, and even among those that are not accredited, a manual review revealed that only $9 \%$ of the nonaccredited Web sites offering information on colorectal cancer would have met HON accreditation standards. The situation is further complicated by which search terms are used, and which language is used for the search (with French having the largest percentage of accredited information).

Compounding all of this confusion is the sheer volume of Web sites devoted to different cancers, from several hundred thousand sites for certain liver and bile duct cancers to 450 million for skin cancer and 461 million for breast cancer. And then there are sponsorship issues, with surgeons/hospitals leading the pack, and nonprofit organizations (who arguably would have the most to gain from providing accurate cancer information and therefore should be considered the most credible by the public) at the bottom of the heap. Just because a website is offered by a health professional or health-related institution/organization does not mean that it contains quality information.

As examples, review of a Web site from a hospital in Atlanta, Georgia, ${ }^{3}$ recommends lung cancer screening for people who do not meet the risk criteria of the recently reported National Lung Screening Trial, ${ }^{4}$ which is considered the best currently available randomized clinical trial to address who benefits from spiral computed tomographic screening in smokers and former smokers. A nationwide prostate cancer organization in the United States ${ }^{5}$ promotes prostate-specific antigen testing for early detection of prostate cancer, with no mention that several reputable organizations question the wisdom of routine prostate cancer screening without informed decision making.

What are the solutions?

The recommendations of the authors are for oncologists and other health professionals to guide patients to "accurate, ethical, and readable health Web sites" and suggest 
that patients should be educated about the value and use of the HON principles and available Web tools to assess compliance with the HON code.

Although it is true that health professionals are viewed by patients as the most accurate providers of health information, efforts to translate this authority to health information contained in Web-based searches and healthrelated Web sites have proven difficult in gaining traction in everyday practice.

The Information RX program provided through the National Library of Medicine ${ }^{6}$ in collaboration with several United States medical professional associations is but one example of such an effort to have physicians direct patients to information that is both accurate and monitored closely for quality. Follow-up studies suggest that such efforts are potentially helpful in focusing physicians on need for health professionals to recommend quality health-related information on the Internet while succeeding in directing patients a reliable source of medical information. Whether those benefits are valid in the long term and truly alter patients' information-seeking behavior remains uncertain. ${ }^{7}$

I wish I could say that I am optimistic that such efforts would prove fruitful, but I must admit to a certain amount of skepticism. With so many demands on the time and attention of physicians and other health professionals involved in providing care to cancer patients throughout the world, I am not certain that they consider quality Internet-based information one of their top priorities. And I am even less optimistic that the authors' recommendation that patients be educated about the HON toolbar, which automatically indicates whether a Web site is HON accredited, will be met with much success.

These recommendations are not without merit. There are many highly regarded organizations, such as the American Cancer Society, who devote considerable resources to providing quality cancer-related information on the Internet. Getting patients to recognize the value of these sites would be beneficial to many, but practical barriers remain. And we haven't even mentioned the issues of language or cultural relevancy, not to ignore the readability of medical information on the Web as it relates to health literacy.

In a world where everyone is an expert and where there are no restraints or limitations on the comments of the universe of patients and others who believe that their experience and knowledge is valid when it comes to information about cancer and its treatment, we are left at the mercy of individuals to make their own assessments about the information they find credible for their personal purposes. That is far from a satisfactory state of affairs, as is well documented in this research. How we as a medical community deal with this issue remains unresolved, the recommendations of the authors notwithstanding.

Perhaps the HON principles-or a similar set of standards offered by other organizations - are indeed the answer. However, getting a practical solution is an ongoing task with an elusive goal. That does not diminish the need to address the problem; rather, it should reinforce our commitment to advancing an effective answer to the dilemma of promoting accurate medical information on the Web.

Continued research such as that reported in this issue of the journal is needed to underscore the enormity of the task and the need to develop successful solutions. ${ }^{1}$ There can be no doubt that we must raise awareness among health professionals that this is a serious problem that requires reasonable and effective answers. Failure to do so may expose patients to unnecessary risks and potentially lifethreatening harm.

\section{REFERENCES}

1. Lawrentschuk N, Sasges D,Tasevski R, Abouassaly R, Scott A, Davis I. Oncology health information quality on the Internet: a multilingual evaluation. Ann Surg Oncol (in press).

2. The HON code of conduct for medical and health. http://www. hon.ch/HONcode/Conduct.html.

3. St. Joseph's Center for Cancer Care and Research: Lung Cancer Screening FAQs. http://www.stjosephsatlanta.org/medical_services/ cancer_care_and_research/ct-lung-screening-frequently\%20askedquestions.html.

4. National Lung Screening Trial Research Team. Reduced lungcancer mortality with low-dose computed tomographic screening. N Engl J Med. 2011;365:395-409.

5. Zero: the project to end prostate cancer. http://zerocancer.org.

6. Information Rx: a free information prescription program from the National Library of Medicine. http://www.informationrx.org.

7. Siegel ER, Logan RA, Harnsberger RL et al. Information Rx: evaluation of a new informatics tool for physicians, patients and libraries. Inf Serv Use. 2006;26:1-10. 